\title{
EDA: EXAFS Data Analysis software package
}

\author{
Alexei KuZmin
}

\begin{abstract}
The EXAFS data analysis software package EDA consists of a suite of programs running under Windows operating system environment and designed to perform all steps of conventional EXAFS data analysis such as the extraction of the XANES/EXAFS parts of the x-ray absorption coefficient, the Fourier filtering, the EXAFS fitting using the Gaussian and cumulant models. Besides, the package includes two advanced approaches, which allow one to reconstruct the radial distribution function (RDF) from EXAFS based on the regularization-like method and to calculate configurationalaveraged EXAFS using a set of atomic configurations obtained from molecular dynamics or Monte Carlo simulations.
\end{abstract}

\section{General concept}

Below the EXAFS data analysis software package EDA (Kuzmin, 1995) is described in details emphasising its key features. The full package, documentation and application examples are available for download at http://www.dragon.lv/eda/.

The EDA package has been under continuous development from 1988. It has been created with an idea to be intuitively simple and fast, guiding the user step by step 
through each part of the EXAFS analysis. Originally developed for the MS-DOS compatible operating systems, the current version of the package consists of a set (Table 1) of interactive programs running under Windows operating system environment. The originality of the EDA package is mainly related to (i) the procedure of the EXAFS oscillation extraction from the experimental data performed by the EDAEES code, (ii) the regularization-like method for the radial distribution function (RDF) reconstruction from EXAFS by the EDARDF code (Kuzmin, 1997) and (iii) the calculation of configuration-averaged EXAFS based on the results of molecular dynamics or Monte Carlo simulations by the EDACA code (Kuzmin \& Evarestov, 2009).

The various components of the EDA package and their relation are shown in Fig. 1, where the main steps of the analysis and the computer codes involved are given. We will describe it briefly below.

When performing XAS experiment, one usually obtains two signals $I_{0}(E)$ and $I(E)$, which are proportional to the intensity of the X-ray beam with the energy $E$ before and after interaction with the sample. These two signals are used to calculated the X-ray absorption coefficient $\mu(E)$ by the EDAFORM code.

At this point, the X-ray absorption near edge structure (XANES) of $\mu(E)$ can be isolated and analysed by calculating its first and second derivatives using the EDAXANES code. This step allows one to determine precisely the position of the absorption edge and, thus, to check the reproducibility of the energy scale for the single sample or to determine the absorption edge shift $\Delta E$ due to a variation of the effective charge of the absorbing atom in different compounds.

The extraction of the EXAFS $\chi(E)=\left(\mu(E)-\mu_{b}(E)-\mu_{0}(E)\right) / \Delta \mu_{0}(E)$ is implemented in the EDAEES code using the following sophisticated procedure. The background contribution $\mu_{b}(E)$ is determined by extrapolating the pre-edge background as $\mu_{b}(E)=A-B / E^{3}$. Next, the atomic-like contribution $\mu_{0}(E)$ is determined as $\mu_{0}(E)=$ 
$\mu_{0}^{I}(E)+\mu_{0}^{I I}(k)+\mu_{0}^{I I I}(k)$, where the three functions $\mu_{0}^{I}(E)=P_{n}(E), \mu_{0}^{I I}(k)=P_{m}(k)$ ( $P_{n}$ is the polynomial of n-order) and $\mu_{0}^{I I I}(k)=S_{3}(k, p)\left(S_{3}(k, p)\right.$ is the smoothing cubic spline with the parameter $p$ ) are calculated in series, the first one in $E$ space whereas the last two in $k$-space ( $k$ is the photoelectron wavenumber). The EXAFS normalization is performed as $\Delta \mu_{0}(E)=\mu_{0}^{I}(E)$. Such procedure guarantees accurate determination of the $\mu_{0}(E)$ function, and as a result, the EXAFS $\chi(k)$, even if experimental data are far from ideal. The $k$ scale is conventionally defined as $k=\sqrt{\left(2 m_{e} / \hbar^{2}\right)\left(E-E_{0}\right)}$, where $m_{e}$ is the electron mass, $\hbar$ is the Planck's constant, and $E_{0}$ is the threshold energy, i.e., the energy of a free electron with zero momentum. Deglitching and normalization of the EXAFS to the edge jump $\Delta \mu_{0}(E)$ obtained from the reference compound, theoretical tables (Teo, 1986) or equal to a constant are also possible.

The extracted experimental EXAFS $\chi(k)$ can be directly compared with the configurationaveraged EXAFS calculated based on the results of molecular dynamics (MD) or Monte Carlo (MC) simulations by the EDACA code or can be analysed in more conventional way. In the latter case, the Fourier filtering procedure (i.e. direct and back Fourier transforms (FTs) with some suitable "window"-function) is applied using the EDAFT code to separate a contribution from the required range in $R$-space into the total EXAFS. Such approach allows one to simplify the analysis, at least, for a contribution from the first coordination shell of the absorbing atom.

Finally, the EXAFS from a single or several coordination shells can be simulated using different models to extract structural information. The EDA package allows one to use three models (the first two will be discussed below): (i) conventional multi-component parameterized model within the Gaussian or cumulant approximation (the EDAFIT code, see Section 2), (ii) arbitrary RDF model determined by the regularization-like approach (the EDARDF code, see Section 3), (iii) the so-called 
"splice" model (Stern et al., 1992) (combined use of the EDAFT and EDAPLOT codes is required). To perform simulations, one needs to provide the scattering amplitude $f(k, R)$ and phase shift $\phi(k, R)$ functions for each scattering path. These data can be obtained from experimental EXAFS spectrum of some reference compound, taken from tables (Teo, 1986; McKale et al., 1988) or calculated theoretically. In the EDA package, one has possibility to use the theoretical data calculated by the FEFF8/9 codes (Ankudinov et al., 1998; Rehr et al., 2010), which can be extracted from the feff****.dat files by the EDAFEFF code.

Finally, the EDAPLOT code is provided for visualization, comparison and simple mathematical analysis of any data obtained within the EDA package. Note that since all data are kept in simple ASCII format, they can be easily imported to and treated by any other codes.

\section{Multi-component model within the Gaussian/cumulant approximation.}

The fitting of the EXAFS $\chi(k)$ in the $k$-space within the single-scattering curved-wave approximation is implemented in the EDAFIT code which is based on the cumulant expansion of the EXAFS equation (Rehr \& Albers, 2000; Kuzmin \& Chaboy, 2014)

$$
\begin{aligned}
\chi(k) & =\sum_{i}^{\text {shells }} S_{0}^{2} \frac{N_{i}}{k R_{i}^{2}} f_{i}\left(\pi, k, R_{i}\right) \exp \left(-2 \sigma_{i}^{2} k^{2}+\frac{2}{3} C_{4 i} k^{4}\right. \\
& \left.-\frac{4}{45} C_{6 i} k^{6}\right) \exp \left(-2 R_{i} / \lambda(k)\right) \sin \left(2 k R_{i}-\frac{4}{3} C_{3 i} k^{3}\right. \\
& \left.+\frac{4}{15} C_{5 i} k^{5}+\phi_{i}\left(\pi, k, R_{i}\right)\right)
\end{aligned}
$$

where $k=\sqrt{k^{\prime 2}+\left(2 m_{e} / \hbar^{2}\right) \Delta E_{0 i}}$ is the photoelectron wavenumber corrected for the difference $\Delta E_{0 i}$ in the energy origin between experiment and theory; $S_{0}^{2}$ is the scale factor taking into account amplitude damping due to multielectron effects; $N_{i}$ is the coordination number of the $\mathrm{i}$-th shell; $R_{i}$ is the radius of the $\mathrm{i}$-th shell; $\sigma_{i}$ is the meansquare relative displacement (MSRD) or Debye-Waller factor; $C_{3 i}, C_{4 i}, C_{5 i}$ and $C_{6 i}$ 
are cumulants of a distribution taking into account anharmonic effects and/or nonGaussian disorder; $\lambda(k)=k / \Gamma$ ( $\Gamma$ is a constant) is the mean free path (MFP) of the photoelectron; $f\left(\pi, k, R_{i}\right)$ is the backscattering amplitude of the photoelectron due to the atoms of the $\mathrm{i}$-th shell; $\phi\left(\pi, k, R_{i}\right)=\psi\left(\pi, k, R_{i}\right)+2 \delta_{l}(k)-l \pi$ is the phase shift containing contributions from the absorber $2 \delta_{l}(k)$ and the backscatterer $\psi\left(\pi, k, R_{i}\right)(l$ is the angular momentum of the photoelectron).

The fitting parameters of the model are $S_{0}^{2} N_{i}, R_{i}, \sigma_{i}^{2}, \Delta E_{0 i}, C_{3 i}, C_{4 i}, C_{5 i}, C_{6 i}$ and $\Gamma$. The maximum number of fitting parameters, which can be used in the EXAFS model, is limited by the Nyquist criterion $N_{\text {par }}=2 \Delta k \Delta R / \pi$ (Stern, 1993).

Note that when the functions $f\left(\pi, k, R_{i}\right)$ and $\phi\left(\pi, k, R_{i}\right)$ are extracted from the EXAFS spectrum of a reference compound, the values of fitting parameters will be relative. To compare different models obtained by fitting of the EXAFS using the EDAFIT code, Fisher's $F_{0.95}$ criterion, implemented in the FTEST code (Kuzmin, 1995), can be applied.

\section{Regularization-like method}

The regularization-like method implemented in the EDARDF code allows one to determine model independent $\operatorname{RDF} G(R)$ from the experimental EXAFS. It is especially suitable for the analysis of the first coordination shell in locally distorted or disordered materials, such as low-symmetry crystals (e.g. with Jahn-Teller distortions), amor-

phous compounds, glasses, and systems with strongly anharmonic behaviour, where a decomposition into the cumulant series fails. At the same time, the method can be also used in more simple cases as a starting point for the selection of a conventional model described in the previous section.

The $\operatorname{RDF} G(R)$ is determined by inversion of the EXAFS equation within the 
single-scattering approximation

$$
\chi(k)=S_{0}^{2} \int_{R_{\min }}^{R_{\max }} \frac{G(R)}{k R^{2}} f(\pi, k, R) \sin (2 k R+\phi(\pi, k, R)) d R
$$

using the iterative method described in (Kuzmin \& Purans, 2000). Two regularizing criteria are applied after each iteration to restrict the shape of $G(R)$ to physically significant solutions: it must be positive-defined and smooth function.

The use of the method is demonstrated in Fig. 2 for the case of tin tungstate, which exists in two phases $-\alpha-\mathrm{SnWO}_{4}$ and $\beta-\mathrm{SnWO}_{4}$ (Kuzmin et al., 2015). In orthorhombic $\alpha-\mathrm{SnWO}_{4}$ phase tungsten atoms are six-fold coordinated by oxygen atoms, and the $\mathrm{WO}_{6}$ octahedra are strongly distorted due to the second-order Jahn-Teller effect because of the $\mathrm{W}^{6+}\left(5 d^{0}\right)$ electronic configuration. The six $\mathrm{W}-\mathrm{O}$ bonds in $\alpha-\mathrm{SnWO}_{4}$ are split into two groups of four short bonds at $\sim 1.82 \AA$ and two long bonds at $\sim 2.15 \AA$. In cubic $\beta-\mathrm{SnWO}_{4}$ tungsten atoms have slightly deformed $\mathrm{WO}_{4}$ tetrahedral coordination with the $\mathrm{W}-\mathrm{O}$ bond lengths of about $1.77 \AA$. An increase of temperature from $10 \mathrm{~K}$ to $300 \mathrm{~K}$ affects weakly the $\mathrm{W}-\mathrm{O}$ bonding in the $\mathrm{WO}_{4}$ tetrahedra and also the group of four shortest $\mathrm{W}-\mathrm{O}$ bonds in the $\mathrm{WO}_{6}$ octahedra. At the same time, the distant group of weakly bound two oxygen atoms in the $\mathrm{WO}_{6}$ octahedra shifts to longer distances and becomes more broadened. Thus, the reconstructed RDFs reproduce nicely the W $\mathrm{L}_{3}$-edge EXAFS in both tin tungstates and allow one to follow a distortion of the tungsten first shell in details.

Another example, shown in Fig. 2, is concerned the local atomic structure relaxation upon crystallite size reduction in $\mathrm{ZnWO}_{4}$ (Kalinko \& Kuzmin, 2011). Crystalline $\mathrm{ZnWO}_{4}$ has monoclinic $(\mathrm{P} 2 / c)$ wolframite-type structure built up of distorted $\mathrm{WO}_{6}$ and $\mathrm{ZnO}_{6}$ octahedra joined by edges into infinite zigzag chains. The distortion of the metal-oxygen octahedra leads to the splitting of the $\mathrm{W}-\mathrm{O}$ and $\mathrm{Zn}-\mathrm{O}$ distances into three groups of two oxygen atoms each with the bond lengths of about 1.79, 1.91 and $2.13 \AA$ around tungsten atoms and 2.03, 2.09 and $2.22 \AA$ around zinc atoms. Note that IUCr macros version 2.1.15: 2021/03/05 
the three $\mathrm{W}-\mathrm{O}$ groups are well resolved in the RDF. Upon the reduction of crystallite size down to $\sim 2 \mathrm{~nm}$, significant relaxation of the atomic structure occurs leading to some broadening of the RDF peaks, especially at large distances (2.1-2.4 $\AA$ ), whereas the nearest oxygen atoms become stronger bound. Such structural changes in $\mathrm{ZnWO}_{4}$ nanoparticles correlate with their optical and vibrational properties.

Other application examples of the method include dehydration process in molybdenum oxide hydrate (Kuzmin \& Purans, 2000), the effect of composition and crystallite size reduction in tungstates (Kuzmin \& Purans, 2001; Anspoks et al., 2014b; Kuzmin et al., 2014), and studies of the local environment in glasses (Kuzmin \& Purans, 1997; Rocca et al., 1998; Rocca et al., 1999; Kuzmin et al., 2006).

\section{Configuration-averaged EXAFS simulations}

A particular feature of the EDA package is its ability to perform more advanced calculations of the configuration-averaged EXAFS based on the results of MD simulations

(Fig. 4) (Kuzmin \& Evarestov, 2009; Kuzmin \& Chaboy, 2014; Kuzmin et al., 2016). Note that a set of atomic configurations generated using the MC simulation (a Beccara \& Fornasini, 2008) can be also used in a similar manner. To use this approach, called MD-EXAFS, one needs to provide an *.XYZ file containing temporal snapshots of atomic coordinates, which can be obtained from most MD codes such as GULP (Gale \& Rohl, 2003), DLPOLY (Todorov et al., 2006), LAMMPS (Plimpton, 1995) or CP2K (VandeVondele et al., 2005). Additionally the input file with a set of commands for the FEFF8/9 code is also required.

A care should be taken to get proper configuration-averaged EXAFS. This means that a number of snapshots should be sufficiently large (usually few thousands) to get good statistics, and the time step between subsequent snapshots should be enough small to sample properly the dynamic properties of the material. The MD-EXAFS IUCr macros version 2.1.15: 2021/03/05 
approach allows one to validate different theoretical models, e.g. force-fields, and/or perform the EXAFS interpretation far beyond the nearest coordination shells.

Application examples of this method cover several compounds: $\mathrm{SrTiO}_{3}$ (Kuzmin \& Evarestov, 2009), $\mathrm{ReO}_{3}$ (Kalinko et al., 2009), Ge (Timoshenko et al., 201), NiO (Anspoks et al., 2010; Anspoks et al., 2012; Anspoks et al., 2014a), $\mathrm{LaCoO}_{3}(\mathrm{Kuzmin}$ et al., 2011), $\mathrm{ZnO}$ (Timoshenko et al., 2014), $\mathrm{AWO}_{4}(\mathrm{~A}=\mathrm{Ca}, \mathrm{Sr}, \mathrm{Ba})$ tungstates (Kalinko et al., 2016), $\mathrm{Y}_{2} \mathrm{O}_{3}$ (Jonane et al., 2016a), $\mathrm{FeF}_{3}$ (Jonane et al., 2016b) and $\mathrm{UO}_{2}$ (Bocharov et al., 2017).

The case of microcrystalline and nanocrystaline (6 nm) NiO (Anspoks et al., 2012) is illustrated in Fig. 5. The Ni K-edge EXAFS spectra of both compounds are dominated by a contribution from the first two coordination shells $\left(\mathrm{Ni}-\mathrm{O}_{1}\right.$ and $\left.\mathrm{Ni}-\mathrm{Ni}_{2}\right)$ of nickel. However, the outer shells are responsible for a number of well resolved peaks located above $\sim 3 \AA$ in the Fourier transforms. Due to cubic rock-salt structure of $\mathrm{NiO}$, the multiple-scattering events play an important role in the formation of EXAFS and must be treated properly. The classical MD simulations (Anspoks et al., 2012) were performed using the force-field model included two-body central force interactions between atoms described by a sum of the Buckingham and Coulomb potentials. The effects of crystallite size, thermal disorder and Ni vacancy concentration were taken into account. The calculated configuration-averaged EXAFS reproduces well the experimental data for both nickel oxides. In the case of nanocrystalline $\mathrm{NiO}$, the damping of the EXAFS oscillations due to the atomic structure relaxation and the progressive decrease of the FT peaks amplitude at longer distances are observed as a result of the crystallite size reduction.

This work was supported by the Latvian Science Council grant no. 187/2012.

\section{References}

IUCr macros version 2.1.15: 2021/03/05 


\section{References}

a Beccara, S. \& Fornasini, P. (2008). Phys. Rev. B, 77, 172304.

Ankudinov, A. L., Ravel, B., Rehr, J. J. \& Conradson, S. D. (1998). Phys. Rev. B, 58, $7565-7576$.

Anspoks, A., Kalinko, A., Kalendarev, R. \& Kuzmin, A. (2012). Phys. Rev. B, 86, 174114.

Anspoks, A., Kalinko, A., Kalendarev, R. \& Kuzmin, A. (2014a). Thin Solid Films, 553, $58-62$.

Anspoks, A., Kalinko, A., Timoshenko, J. \& Kuzmin, A. (2014b). Solid State Commun. 183, $22-26$.

Anspoks, A., Kuzmin, A., Kalinko, A. \& Timoshenko, J. (2010). Solid State Commun. 150, 2270-2274.

Bocharov, D., Chollet, M., Krack, M., Bertsch, J., Grolimund, D., Martin, M., Kuzmin, A., Purans, J. \& Kotomin, E. (2017). Prog. Nucl. Energy, 94, 187-193.

Gale, J. D. \& Rohl, A. L. (2003). Mol. Simul. 29, 291-341.

Jonane, I., Lazdins, K., Timoshenko, J., Kuzmin, A., Purans, J., Vladimirov, P., Gräning, T. \& Hoffmann, J. (2016a). J. Synchrotron Rad. 23, 510-518.

Jonane, I., Timoshenko, J. \& Kuzmin, A. (2016b). Phys. Scr. 91, 104001.

Kalinko, A., Bauer, M., Timoshenko, J. \& Kuzmin, A. (2016). Phys. Scr. 91, 114001.

Kalinko, A., Evarestov, R., Kuzmin, A. \& Purans, J. (2009). J. Phys.: Conf. Series, 190, 012080 .

Kalinko, A. \& Kuzmin, A. (2011). J. Non-Cryst. Solids, 357, 2595-2599.

Kuzmin, A. (1995). Physica B, 208-209, 175-176.

Kuzmin, A. (1997). J. Physique IV (France), 7, C2-213-C2-214.

Kuzmin, A., Anspoks, A., Kalinko, A. \& Timoshenko, J. (2016). Z. Phys. Chem. 230, 537-549.

Kuzmin, A., Anspoks, A., Kalinko, A., Timoshenko, J. \& Kalendarev, R. (2014). Phys. Scr. 89, 044005 .

Kuzmin, A., Anspoks, A., Kalinko, A., Timoshenko, J. \& Kalendarev, R. (2015). Sol. Energy Mater. Sol. Cells, 143, 627-634.

Kuzmin, A. \& Chaboy, J. (2014). IUCrJ, 1, 571-589.

Kuzmin, A., Dalba, G., Fornasini, P., Rocca, F. \& Šipr, O. (2006). Phys. Rev. B, 73, 174110.

Kuzmin, A., Efimov, V., Efimova, E., Sikolenko, V., Pascarelli, S. \& Troyanchuk, I. O. (2011). Solid State Ionics, 188, 21-24.

Kuzmin, A. \& Evarestov, R. A. (2009). J. Phys.: Condens. Matter, 21, 055401.

Kuzmin, A. \& Purans, J. (1997). Proc. SPIE, 2968, 180-185.

Kuzmin, A. \& Purans, J. (2000). J. Phys.: Condens. Matter, 12, 1959-1970.

Kuzmin, A. \& Purans, J. (2001). Rad. Meas. 33, 583-586.

McKale, A. G., Veal, B. W., Paulikas, A. P., Chan, S. K. \& Knapp, G. S. (1988). J. Am. Chem. Soc. 110, 3763-3768.

Plimpton, S. (1995). J. Comp. Phys. 117, 1-19.

Rehr, J. J. \& Albers, R. C. (2000). Rev. Mod. Phys. 72, 621-654.

Rehr, J. J., Kas, J. J., Vila, F. D., Prange, M. P. \& Jorissen, K. (2010). Phys. Chem. Chem. Phys. 12, 5503-5513.

Rocca, F., Dalba, G., Grisenti, R., Bettinelli, M., Monti, F. \& Kuzmin, A. (1998). J. NonCryst. Solids, 232-234, 581-586.

Rocca, F., Dalmasso, A., Monti, F., Kuzmin, A. \& Pasqualini, D. (1999). J. Synchrotron Rad. 6, $737-739$.

Stern, E. A. (1993). Phys. Rev. B, 48, 9825-9827.

Stern, E. A., Ma, Y., Hanske-Petitpierre, O. \& Bouldin, C. E. (1992). Phys. Rev. B, 46, 687-694.

Teo, B. K. (1986). EXAFS: Basic Principles and Data Analysis. Berlin: Springer.

IUCr macros version 2.1.15: 2021/03/05 
Timoshenko, J., Anspoks, A., Kalinko, A. \& Kuzmin, A. (2014). Acta Mater. 79, 194-202.

Timoshenko, J., Kuzmin, A. \& Purans, J. (201). Centr. Eur. J. Phys. 9, 710-715.

Todorov, I. T., Smith, W., Trachenko, K. \& Dove, M. T. (2006). J. Mater. Chem. 16, 19111918.

VandeVondele, J., Krack, M., Mohamed, F., Parrinello, M., Chassaing, T. \& Hutter, J. (2005). Comp. Phys. Commun. 167, 103-128.

Table 1. A set of programs for EXAFS data analysis and simulations included in the EDA software

\begin{tabular}{|c|c|}
\hline Code title & Code description \\
\hline EDAFORM & $\begin{array}{l}\text { converts original experimental data from several beamlines into } \\
\text { the EDA file format (ASCII, } 2 \text { columns). }\end{array}$ \\
\hline EDAXANES & $\begin{array}{l}\text { extracts the XANES part of the experimental X-ray absorption } \\
\text { spectrum and calculates its first and second derivatives. }\end{array}$ \\
\hline EDAEES & $\begin{array}{l}\text { extracts the EXAFS part } \chi(k) \text { using original algorithm for the } \\
\text { atomic-like ("zero-line") background removal. }\end{array}$ \\
\hline EDAFT & $\begin{array}{l}\text { performs Fourier filtering procedure (direct and back Fourier } \\
\text { transforms) with or without amplitude/phase correction using } \\
\text { a number of different (rectangular, Gaussian, Kaiser-Bessel, } \\
\text { Hamming and Norton-Beer F3) window functions. }\end{array}$ \\
\hline EDAFIT & $\begin{array}{l}\text { is a non-linear least-squares fitting code, based on a high speed } \\
\text { algorithm without matrix inversion. A multi-shell Gaussian or } \\
\text { cumulant model within the single-scattering approximation can } \\
\text { contain up to } 20 \text { shells with up to } 8 \text { fitting parameters }\left(N_{i}, S_{o}^{2} \text {, }\right. \\
\left.R_{i}, \sigma_{i}^{2}, \Delta E_{0 i}, C_{3 i}, C_{4 i}, C_{5 i}, C_{6 i}\right) \text { in each. Constrains on the range } \\
\text { of any fitting parameter or its value can be imposed. }\end{array}$ \\
\hline EDARDF & $\begin{array}{l}\text { is the regularization-like least-squares-fitting code allowing one } \\
\text { to determine model-independent RDF in the first coordination } \\
\text { shell for a compound with arbitrary degree of disorder. }\end{array}$ \\
\hline FTEST & $\begin{array}{l}\text { performs analysis of variance of the fitting results based on } \\
\text { the Fisher's } F_{0.95} \text {-test. }\end{array}$ \\
\hline EDAPLOT & $\begin{array}{l}\text { is a general-purpose program for plotting, comparison, and } \\
\text { mathematical calculations frequently used in the EXAFS data } \\
\text { analysis (more than } 20 \text { different operations). }\end{array}$ \\
\hline EDAFEFF & $\begin{array}{l}\text { extracts the scattering amplitude and phase shift functions from } \\
\text { FEFF****.dat files, calculated by the FEFF } 8 / 9 \text { code, for the use } \\
\text { with the EDAFIT or EDARDF codes. }\end{array}$ \\
\hline EDACA & $\begin{array}{l}\text { calculates configuration-averaged EXAFS based on the results } \\
\text { of molecular dynamics simulations. }\end{array}$ \\
\hline
\end{tabular}




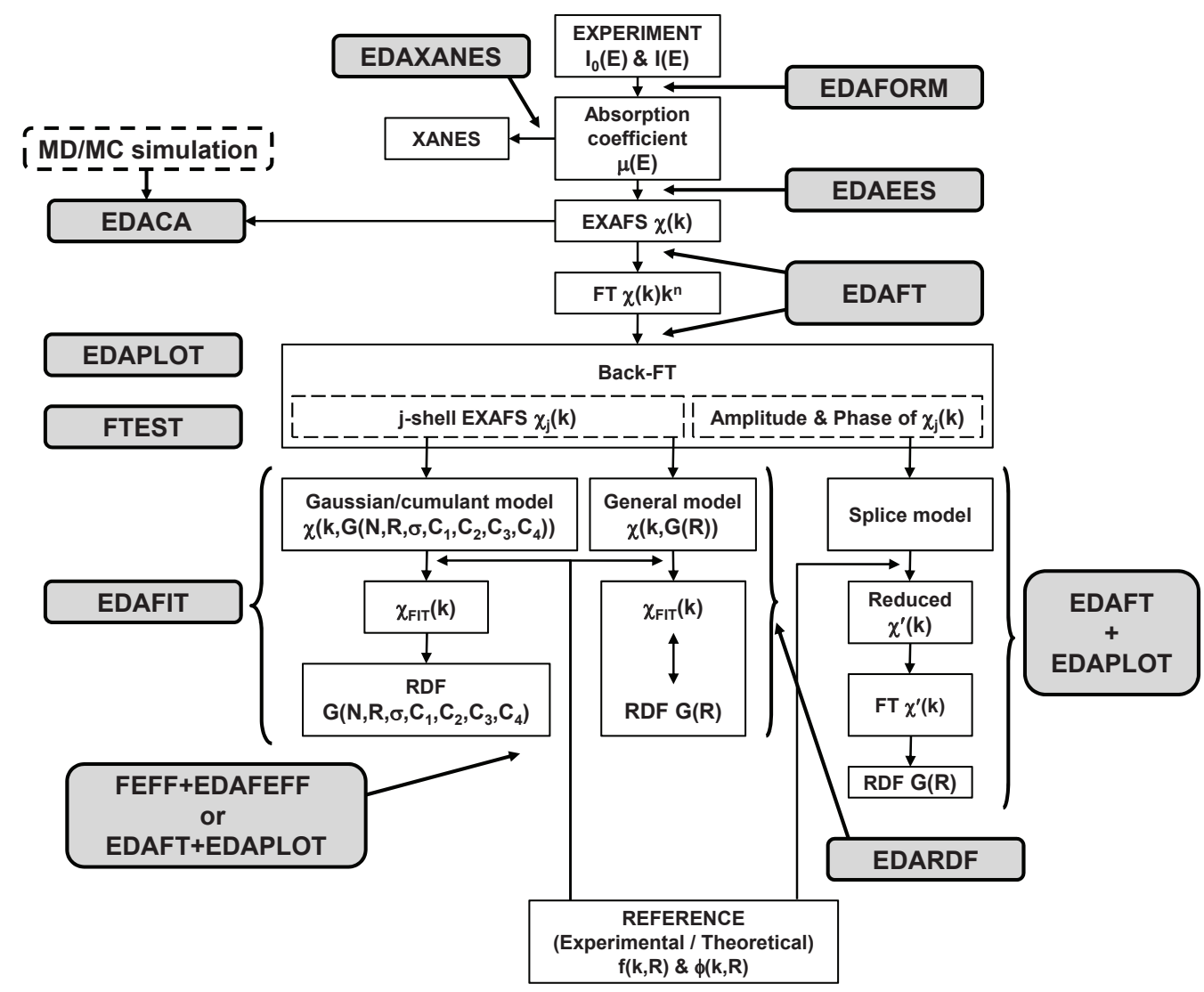

Fig. 1. Flowchart of the EXAFS data analysis by the EDA package. 

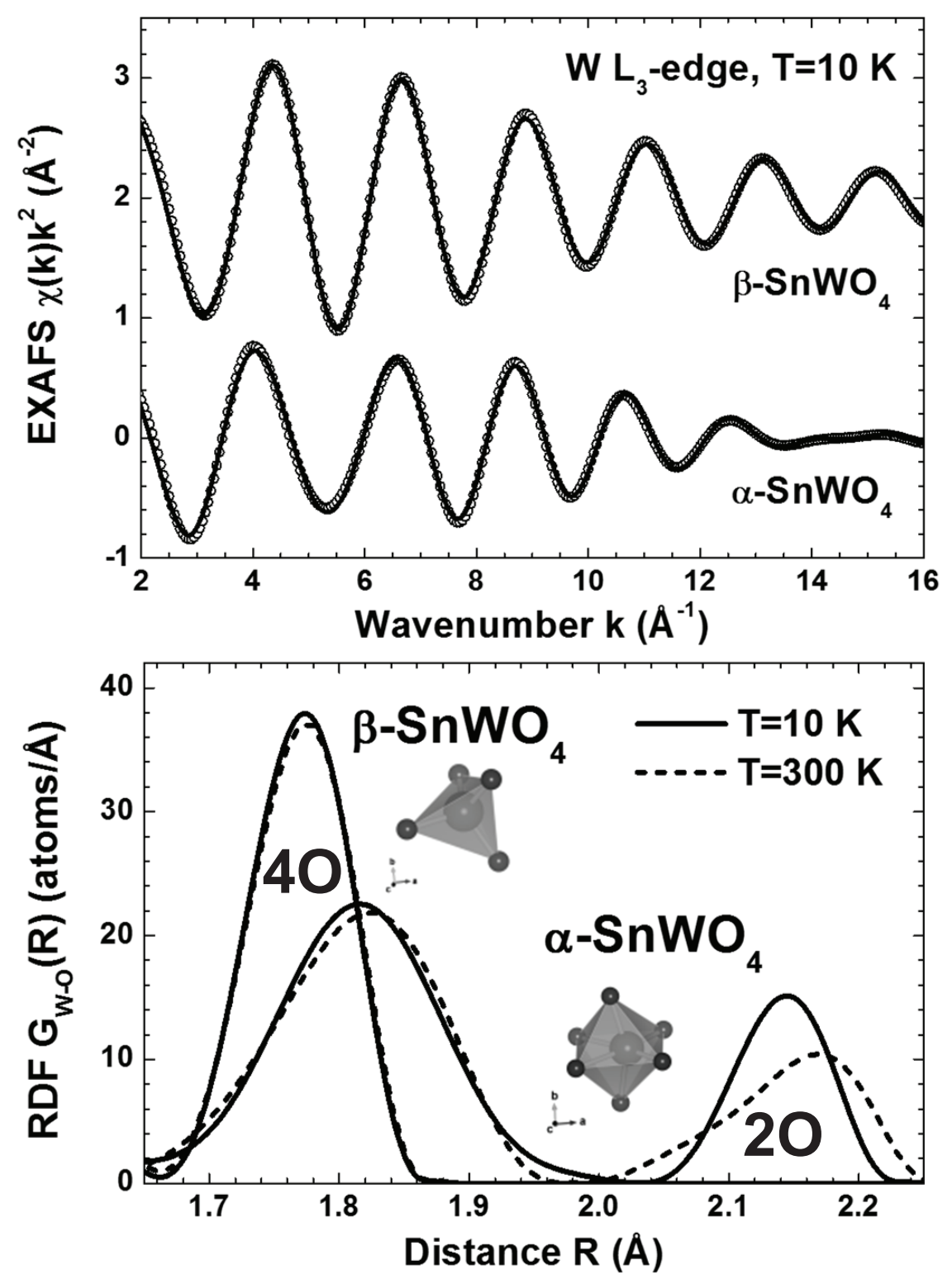

Fig. 2. Upper panel: comparison of the experimental (circles) and calculated (solid lines) $\mathrm{W} \mathrm{L}_{3}$-edge EXAFS spectra $\chi(k) k^{2}$ for the first coordination shell of tungsten in $\alpha-\mathrm{SnWO}_{4}$ (lower curves) and $\beta$-SnWO 4 (upper curves) at $10 \mathrm{~K}$. Lower panel: calculated radial distribution functions (RDF's) $G_{\mathrm{W}-\mathrm{O}}(R)$ for $\mathrm{W}-\mathrm{O}$ bonds within the first coordination shell of tungsten in $\alpha-\mathrm{SnWO}_{4}$ and $\beta-\mathrm{SnWO}_{4}$ at $10 \mathrm{~K}$ (solid lines) and $300 \mathrm{~K}$ (dashed lines). The two groups of 4 and 2 oxygen atoms are indicated. 


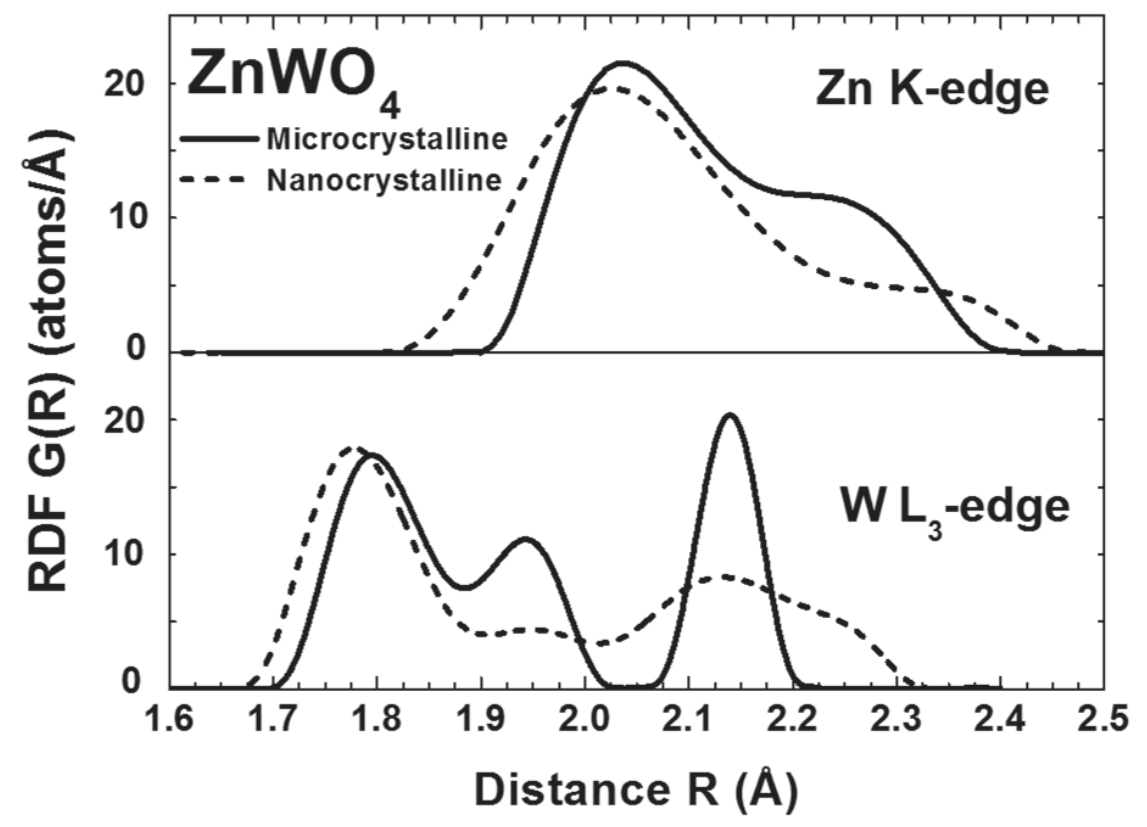

Fig. 3. The reconstructed RDFs $G(R)$ for the first coordination shell of tungsten and zinc in nanoparticles and microcrystalline $\mathrm{ZnWO}_{4}$. 


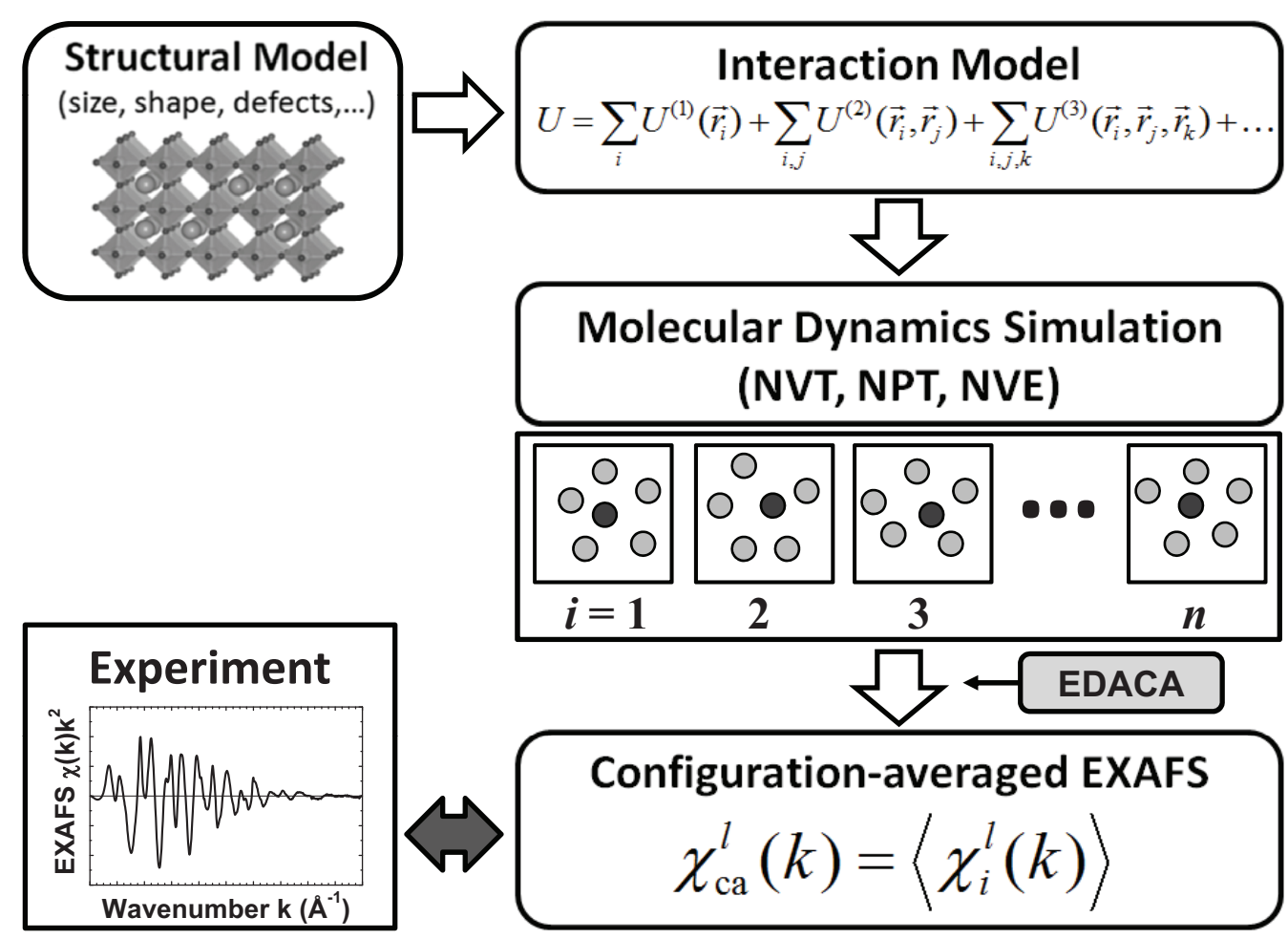

Fig. 4. Flowchart of the MD-EXAFS calculations. 

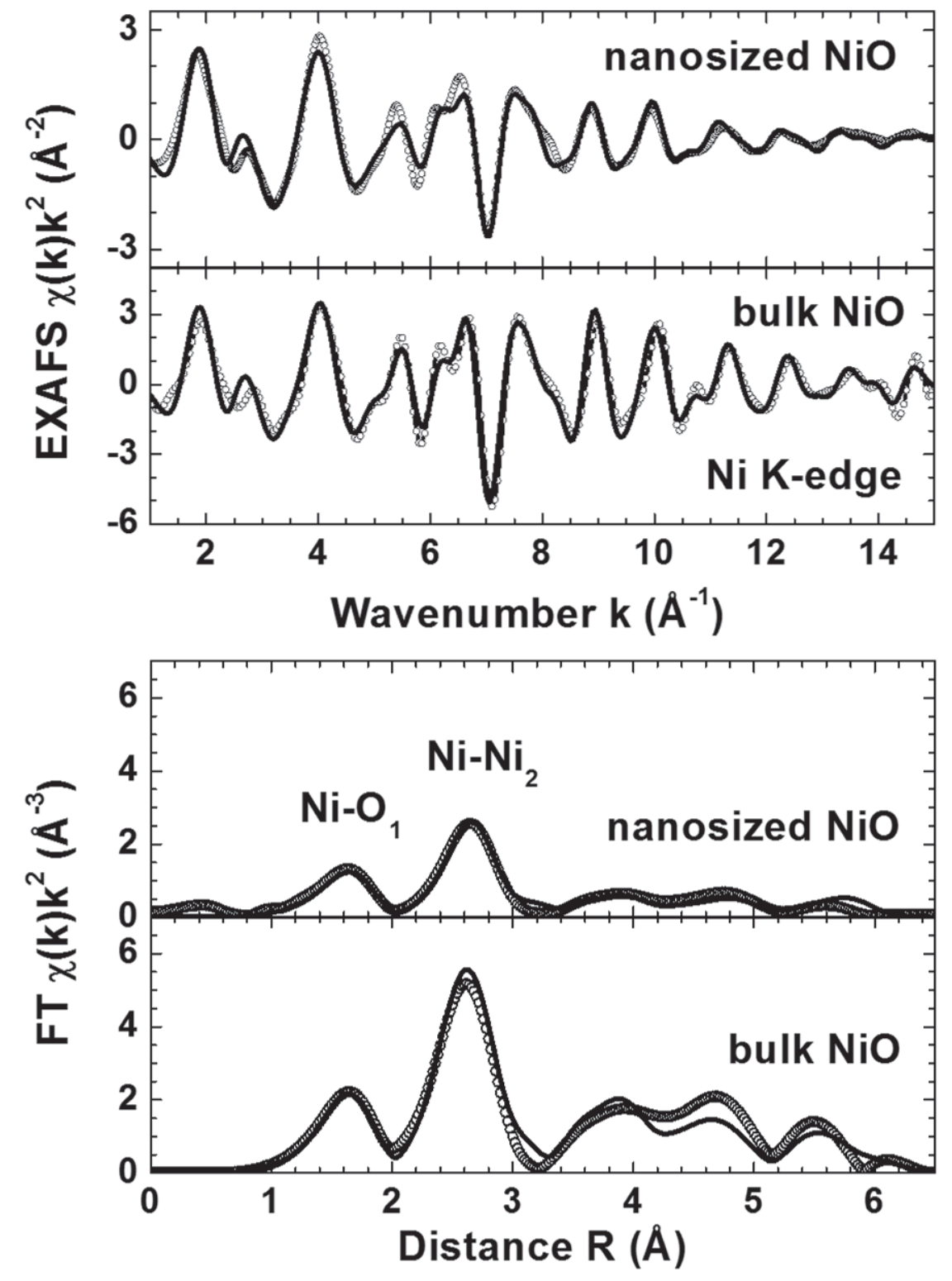

Fig. 5. Comparison of the experimental (circles) and calculated configuration-averaged (solid lines) Ni K-edge EXAFS spectra $\chi(k) k^{2}$ (upper panel) and their Fourier transforms (lower panel) for bulk and nanosized $\mathrm{NiO}$ at $300 \mathrm{~K}$. 